\title{
Article
}

\section{Acute effects of knee wraps/ sleeve on kinetics, kinematics and muscle forces during the barbell back squat}

Sinclair, Jonathan Kenneth, Mann, James, Weston, George, Poulsen, Nik, Edmundson, Christopher James, Bentley, Ian and Stone, Mark

Available at http://clok.uclan.ac.uk/29987/

Sinclair, Jonathan Kenneth ORCID: 0000-0002-2231-3732, Mann, James, Weston, George, Poulsen, Nik, Edmundson, Christopher James ORCID: 00000003-2275-7584, Bentley, Ian ORCID: 0000-0002-9086-2338 and Stone, Mark ORCID: 0000-0003-0735-287X (2019) Acute effects of knee wraps/ sleeve on kinetics, kinematics and muscle forces during the barbell back squat. Sport Sciences for Health, 16 . pp. 227-237. ISSN 1824-7490

It is advisable to refer to the publisher's version if you intend to cite from the work. http://dx.doi.org/10.1007/s11332-019-00595-5

For more information about UCLan's research in this area go to

http://www.uclan.ac.uk/researchgroups/ and search for <name of research Group>.

For information about Research generally at UCLan please go to http://www.uclan.ac.uk/research/

All outputs in CLoK are protected by Intellectual Property Rights law, including Copyright law. Copyright, IPR and Moral Rights for the works on this site are retained by the individual authors and/or other copyright owners. Terms and conditions for use of this material are defined in the policies page. 


\section{Acute effects of knee wraps/ sleeve on kinetics, kinematics and muscle forces during the}

\section{barbell back squat.}

3 Jonathan Sinclair ${ }^{1}$, James Mann ${ }^{1}$, George Weston ${ }^{1}$, Nikolaj Poulsen ${ }^{1}$, Christopher James

4 Edmundson ${ }^{1}$, Ian Bentley ${ }^{1} \&$ Mark Stone ${ }^{1}$

5 1. Centre for Applied Sport and Exercise Sciences, Faculty of Health and Wellbeing,

6 University of Central Lancashire, Lancashire, Preston, Lancashire, United Kingdom.

7

8 Correspondence Address:

9 Dr. Jonathan Sinclair

10 Centre for Applied Sport \& Exercise Sciences

11 Faculty of Health and Wellbeing

12 University of Central Lancashire

13 Preston

14 Lancashire

15 PR1 2HE.

16 E-mail: jksinclair@uclan.ac.uk

17 Keywords: Biomechanics; knee wraps; squat; kinetics; kinematics. 
PURPOSE: The aim of the current investigation was to comparatively examine the effects of knee wraps/ sleeves on kinetics, three-dimensional kinematics and muscle forces during the barbell back squat. METHODS: Fifteen male lifters completed squats at $70 \%$ of their 1 repetition maximum, in four different conditions (nothing, competition knee wrap, training knee wrap and knee sleeve). Three-dimensional kinematics were measured using an eightcamera motion analysis system, ground reaction forces (GRF) using a force platform and muscle forces using musculoskeletal modelling techniques. Differences between conditions were examined using one-way repeated measures ANOVA. RESULTS: The results showed that the integral of the quadriceps (nothing=58.30, competition=51.87 \& training wrap $=53.33 \mathrm{~N} / \mathrm{kg} \cdot \mathrm{s}), \quad$ hamstring $\quad$ (nothing=39.01, competition=35.61 \& training wrap $=33.97 \mathrm{~N} / \mathrm{kg} \cdot \mathrm{s}$ ), gluteus maximus (nothing $=24.29$, competition=22.22 \& training wrap $=21.03 \mathrm{~N} / \mathrm{kg} \cdot \mathrm{s}), \quad$ gastrocnemius $\quad$ (nothing=7.25, competition=5.97 \& training wrap $=6.39 \mathrm{~N} / \mathrm{kg} \cdot \mathrm{s}$ ) and soleus muscles (nothing $=15.49$, competition $=12.75 \&$ training wrap $=13.64 \mathrm{~N} / \mathrm{kg} \cdot \mathrm{s}$ ) during the ascent phase was significantly greater in the nothing condition compared to both knee wraps. In addition, whilst knee wraps and knee sleeves significantly improved perceived knee stability, perceived comfort was significantly reduced in the knee wraps and improved in the knee sleeve. CONCLUSIONS: Taking into account the reduced muscle kinetics, knee wraps may diminish lower extremity muscle development. Therefore, knee sleeves may be more efficacious for athletes who regularly utilize the back squat for their training goals, although further longitudinal analyses are required before this can be fully established. 
43 The back squat is perhaps the most frequently utilized resistance training exercise (1).

44 Because of its ability to recruit the quadriceps, gluteal, hamstrings, tibialis anterior, triceps

45

46

47 surae and lumbar muscles (2), it forms the basis of most strength and conditioning regimens (3).

Because heavy loads are typically borne during the back squat exercise, many athletes choose to perform their squat activities using external supports (4). Knee wraps and knee sleeves are commonly adopted by those involved in competitive and recreational resistance training (5). As described by Lake et al., (3), knee wraps are typically made from thick canvas with interwoven rubber filaments to provide elasticity. To be compliant with International Powerlifting Federation (IPF) regulations, knee wraps can be a maximum of $2 \mathrm{~m}$ in length and should be wrapped as tightly around the knee as possible (3). Similarly, knee sleeves are characteristically made from a dense yet elasticated material such as neoprene in order to provide both elasticity and durability. To be compliant with International Powerlifting Federation (IPF) regulations, knee sleeves can be a maximum of $0.3 \mathrm{~m}$ in length and should provide a high level of compression around the knee joint.

Knee wraps and sleeves are utilized to mediate a mechanical advantage during the back squat exercise (5). They are adopted by both competitive and recreational lifters in order to enhance performance during the squat exercise (3). During the eccentric (descent) phase of the back squat, the knee joint exhibits active flexion in order to lower the bar, allowing the elastic material which comprises the knee wrap/ sleeve to deform (6). When the device is deformed, elastic energy is stored within the bonds between the atoms that make up the sleeve/ wrap. 
66 This potential energy is released as kinetic energy during the concentric (ascent) phase of the

67 lift, in a process known in strength \& conditioning literature as carryover (6).

There has been surprisingly little research concerning the influence of knee wraps/ sleeves on the biomechanics of the squat. Lake et al., (3) examined the effects of knee wraps on biomechanical and performance parameters at $80 \%$ of 1 repetition max (1RM) during the barbell back squat. Their findings showed that horizontal bar displacement was significantly reduced, the lowering phase was performed significantly faster and peak power was significantly greater when wearing knee wraps. This led Lake et al., (3) to conclude that knee wraps enhanced mechanical output but altered the squat technique in a manner that may affect the target musculature and possibly diminish the integrity of the knee joint. Gomes et al., (6) examined the effects of knee wraps on muscle activation (EMG) and joint kinematics at 60 and $90 \%$ of back squat 1RM. Their findings showed that vastus lateralis activation was significantly greater at $60 \% 1 \mathrm{RM}$ but significantly reduced at $90 \% 1 \mathrm{RM}$ when wearing knee wraps. There was also a significant increase in gluteus maximus muscle activity when wearing knee wraps but only at $60 \% 1 \mathrm{RM}$, and a significant increase in peak knee flexion at both 60 and 90\% 1RM. Gomes et al., (5) examined the effects of hard and soft knee wraps on the peak vertical ground reaction force (GRF) produced during an isometric squat. This study showed that peak vertical GRF was significantly greater in both hard and soft knee wraps compared to performing without wraps. Finally, Marchetti et al., (4) analysed the influence of two different techniques of knee wraps placement (spiral where the wrap is placed on the knee in a circular fashion and $\mathrm{X}$ where the wrap is placed in a crossover fashion) on peak vertical GRF and rating of perceived exertion during an isometric barbell back squat. Their findings showed that although peak vertical GRF was greater in both techniques compared to performing without knee wraps, there were no differences between spiral and X conditions. 
92 Despite the aforementioned scientific outputs concerning the effects of knee wraps/ sleeves on the biomechanics of the barbell back squat, there has yet to be any scientific investigation

94 that has concomitantly examined the effects of knee wraps/ sleeves on the kinetics, three-

95 dimensional kinematics and muscle forces of the barbell back squat. Therefore, such an 96 investigation may provide further insight regarding the effects of knee wraps/ sleeves on 97 biomechanical outcomes during the barbell back squat. As such, the aim of the current 98 investigation was to comparatively examine the effects of knee wraps/ sleeves on kinetics, 99 three-dimensional kinematics and muscle forces during the squat.

\section{Methods}

\section{Participants}

103

Fifteen male (age: $23.00 \pm 3.47$ years, stature: $181.93 \pm 7.25 \mathrm{~cm}$, mass: $85.83 \pm 17.10 \mathrm{~kg}$ and 1RM back squat: $122.62 \pm 24.43 \mathrm{~kg}$ ) participants took part in the current study. Participants were all practiced in the high bar back squat with a minimum of 2 years of experience in this lift. All were free from musculoskeletal pathology at the time of data collection and provided written informed consent. All procedures performed were in accordance with the ethical

Helsinki declaration. 
112 Four experimental conditions were examined as part of the current investigation; nothing,

113 knee sleeve, competition wrap and training wrap. The knee sleeve (Strength Shop, Inferno),

114 was made of Neoprene with a thickness of $0.007 \mathrm{~m}$ and length of $0.30 \mathrm{~m}$ in line with IPF

115 regulations. The sleeve came in four different sizes; small, medium, large and extra-large to

116 accommodate all participants. The competition (SBD apparel, Knee Wraps, Competition) and

117 training (SBD apparel, Knee Wraps, Training) wraps had a length of $2 \mathrm{~m}$ and width of $0.08 \mathrm{~m}$

118 in compliance with IPF regulations. The same researcher positioned the knee wraps as tightly

119 as possible before each trial. After completion of their data collection, in accordance with

120 Sinclair et al., (7), each participant subjectively rated each sleeve/ wrap in relation to

121 performing in the nothing condition in terms of stability and comfort. This was accomplished using 3 point scales that ranged from $1=$ improved comfort, $2=$ no change and $3=$ reduced comfort and $1=$ improved stability, $2=$ no change and $3=$ decreased stability. Finally, the participants were also asked to subjectively indicate which of the four conditions that they preferred to perform their squat activities in.

Procedure

128 Three-dimensional kinematics were captured using an eight-camera motion analysis system 129 (Qualisys Medical AB, Goteburg, Sweden) which sampled at $250 \mathrm{~Hz}$. In addition, to capture 130 GRF data piezoelectric force plates (Kistler, Kistler Instruments Ltd., Alton, Hampshire) were adopted, which collected data at $1000 \mathrm{~Hz}$. Kinematics and GRF information were synchronously collected using an analogue to digital interface board. 
134 Body extremity segments were modelled in 6 degrees of freedom using the calibrated anatomical systems technique (8), using a marker configuration utilized previously to quantify the biomechanics of the squat (9). The anatomical frames of the torso, pelvis, thighs, shanks and feet were delineated via the retroreflective markers described by Sinclair et al., (9). Carbon-fiber tracking clusters comprising of four non-linear retroreflective markers were positioned onto the thigh and shank segments. In addition to these the foot segments were tracked via the calcaneus, first metatarsal and fifth metatarsal, the pelvic segment using the PSIS and ASIS markers and the torso via C7, T12 and xiphoid process. Finally, a further two markers were positioned at either end of the bar. The centres of the ankle and knee joints were delineated as the mid-point between the malleoli and femoral epicondyle markers (10, 11), whereas the hip joint centre was obtained using the positions of the ASIS markers (12).

Static calibration trials (not normalized to static trial posture) were obtained with the participant in the anatomical position in order for the positions of the anatomical markers to be referenced in relation to the tracking clusters/markers. A static trial was conducted with the participant in the anatomical position in order for the anatomical positions to be referenced in relation to the tracking markers, following which those not required for dynamic data were removed. The $\mathrm{Z}$ (transverse) axis was oriented vertically from the distal segment end to the proximal segment end. The Y (coronal) axis was oriented in the segment from posterior to anterior. Finally, the $\mathrm{X}$ (sagittal) axis orientation was determined using the right-hand rule and was oriented from medial to lateral.

\section{Squat protocol}


157 For data collection, all participants presented to the laboratory 48 hours after their previous 158 lower-body resistance training session. Before the measured squats were initiated, a general 159 warm up was completed, followed by squat warm-up sets with 30 and $50 \%$ of 1RM (13). 160 Participants completed five continuous high bar back squat repetitions at $70 \%$ of their 1RM, 161 in each if the four experimental conditions using a counterbalanced order. Participants reported their 1RM in the absence of wraps/ sleeves, as the aim was to delineate the maximum squat capacity without aid. A rest period of 3 minutes was enforced between each

lift (3). A load of 70\% of 1RM was selected in accordance with Sinclair et al., (14) and was deemed to be representative of a typical training load, whilst still maintaining the levels of repeatability necessary obtain a representative data set. In accordance with the NSCA guidelines, lifters were instructed to descend in a controlled manner to femur parallel, keep

both feet flat on the floor, preserve proper breath control and maintain a constant/ stable pattern of motion for each repetition. Each participant was examined visually by an NSCA certified strength and conditioning specialist.

Processing

Marker trajectories were digitized using Qualisys Track Manager and then exported as C3D files. Kinematic parameters were quantified using Visual 3-D (C-Motion Inc, Gaithersburg, USA). Marker data was smoothed using a low-pass Butterworth 4th order zero-lag filter at a cut off frequency of $6 \mathrm{~Hz}(15)$. Kinematics of the hip, knee, ankle and trunk were quantified using an XYZ cardan sequence of rotations and joint moments using newton-euler inverse dynamics. All data were normalized to $100 \%$ of the squat via the first and second instances of maximal hip flexion (15). A further time point at the mid-point of the lift that separated the 
dimensional kinematic measures from the hip, knee, ankle which were extracted for statistical analysis were 1) peak angle and 2) angular range of motion (ROM) from initiation to peak angle. In addition, sagittal plane measures from the trunk of 1) peak angle and 2) angular range of motion $(\mathrm{ROM})$ were extracted. In addition to the above, the maximum velocity $(\mathrm{m} / \mathrm{s})$ of the barbell during the ascent phase was quantified, as was the maximum anterior displacement $(\mathrm{m})$ of the barbell during the squat movement.

Quadriceps force was calculated using a musculoskeletal model (16). The quadriceps force was resolved by dividing the knee flexor moment from inverse-dynamics by the moment arm of the quadriceps muscle. The moment arm of the quadriceps was calculated by fitting a 2 nd order polynomial curve to the knee flexion angle-quadriceps moment arm data presented by van Eijden et al., (16).

Hamstring, gluteus maximus, soleus and gastrocnemius forces were also quantified using musculoskeletal modelling approaches (17). The hamstring and gluteus maximus forces were calculated firstly using the hip extensor moment from inverse-dynamics and the hamstrings and gluteus maximus cross-sectional areas, which determined the extent of the joint moment attributable to each muscle (18). The hamstring muscle forces were then calculated by dividing the hip extensor moment attributable to each muscle by the muscle moment arms (19). The moment arms were obtained by fitting a 2 nd order polynomial curve to the hip flexion angle-hamstrings/ gluteus maximus moment arm data of Nemeth \& Ohlsen, (19). In addition, the gastrocnemius and soleus forces were calculated firstly by quantifying the ankle plantarflexor force, which was resolved by dividing the dorsiflexion moment from inverse dynamics by the Achilles tendon moment arm. The Achilles tendon moment arm was 
and soleus muscles was calculated via the cross-sectional area of this muscle relative to the

total volume of the triceps-surae (18).

All muscle forces were normalized by dividing the net values by body mass (N/kg). From the above processing, peak quadriceps, hamstring, gluteus maximus soleus and gastrocnemius forces were extracted for statistical analysis. In addition, the integral of these forces $(\mathrm{N} / \mathrm{kg} \cdot \mathrm{s})$ were calculated during the ascent and descent phases using a trapezoidal function. Finally, the peak rate of force development (RFD) at each of the quadriceps, hamstring, gluteus maximus soleus and gastrocnemius muscles during the ascent phase was also extracted by obtaining the peak increase in muscle force between adjacent data points using the first derivative function within Visual 3D (N/kg/s).

The maximum extent to which the knee joint centre moved anteriorly and laterally during the squat movement (m) was also calculated using Visual 3D. In addition, internal knee joint forces were also calculated in accordance with using the joint force function within Visual 3D et al., (22). The knee flexion moment quantified using inverse dynamics was divided by the moment arm of the patellar tendon. The tendon moment arm was quantified by fitting a 2 nd order polynomial curve to the knee flexion angle-patellar tendon moment arm data provided

by Herzog \& Read, (23). Patellofemoral stress was also quantified by dividing the 
al., (16). Patellofemoral contact areas were obtained by fitting a 2 nd order polynomial curve

[eq1] constant $=\left(0.462+0.00147 *\right.$ knee flexion angle ${ }^{2}-0.0000384 *$ knee flexion angle

\section{$\left.{ }^{2}\right) /\left(1-0.0162 *\right.$ knee flexion angle $+0.000155 *$ knee flexion angle ${ }^{2}-0.000000698 *$}

\section{knee flexion angle ${ }^{3}$ )}

The peak knee joint shear force, patellar tendon force, patellofemoral force $(\mathrm{N} / \mathrm{kg})$ and patellofemoral stress $(\mathrm{KPa} / \mathrm{kg})$ were extracted following normalization to body mass. The instantaneous loading rate of the aforementioned knee force $(\mathrm{N} / \mathrm{kg} / \mathrm{s})$ and stress $(\mathrm{KPa} / \mathrm{kg} / \mathrm{s})$ parameters was calculated by obtaining the peak increase force/ stress between adjacent data points using the first derivative function within Visual 3D. In addition, the integral of the aforementioned parameters $(\mathrm{N} / \mathrm{kg} \cdot \mathrm{s}$ and $\mathrm{KPa} / \mathrm{kg} \cdot \mathrm{s})$ were calculated during the entire squat movement using a trapezoidal function.

244 From the force plate, peak vertical GRF (N/kg) during the ascent phase of the lift was extracted. The RFD of the vertical GRF (N/kg/s) was also calculated by obtaining the peak increase in vertical GRF force between adjacent data points again using the first derivative

247 function within Visual 3D. In addition, the integral of the vertical, medio-lateral anterioposterior GRF's (N/kg.s) were calculated during both the ascent and descent phases of the lift, again using a trapezoidal function. Furthermore, the peak power applied to the centre of mass $(\mathrm{W} / \mathrm{kg})$ during ascent phase was extracted using a product of the vertical GRF and the 
252 also calculated using the time difference from the initiation to the end of each repetition, and 253 the absolute duration of the ascent/ descent phases (s) was also extracted as was the \% 254 duration of the ascent/ descent phases, which were expressed as a function of the total lift 255 duration.

Descriptive statistics of means and standard deviations were obtained for each outcome measure. Shapiro-Wilk tests were used to screen the data for normality. Differences in biomechanical parameters between each of the four conditions were examined using one-way repeated measures ANOVA's. Effect sizes were calculated using partial eta ${ }^{2}\left(\mathrm{p}^{2}\right)$. Effect significant main effect, post-hoc pairwise comparisons were conducted. In addition, the data from participants' subjective ratings in relation to their preferred condition and also in regards to the stability and comfort of each sleeve/ wrap were explored using Chi-Square $\left(X^{2}\right)$ tests. Statistical actions were conducted using SPSS v25.0 (SPSS Inc., Chicago, USA) and Statistical significance was accepted at the $\mathrm{P} \leq 0.05$ level.

\section{Results}

\section{Kinetic and temporal parameters}

271 There was a significant main effect for the integral of the vertical GRF during the descent

272 phase $\left(\mathrm{P} \leq 0.05, \mathrm{p}^{2}=0.19\right)$. Post-hoc pairwise comparisons showed that the vertical GRF 273 integral was significantly greater in the knee sleeve compared to the nothing condition $274(\mathrm{P}=0.01)$ and in the competition wrap in relation to the knee sleeve $(\mathrm{P}=0.036)$. There was also 
a main effect for the extent of anterior bar displacement $\left(\mathrm{P} \leq 0.05, \mathrm{p} \eta^{2}=0.25\right)$. Post-hoc pairwise comparisons showed that bar displacement was significantly greater in the nothing condition compared to the competition $(\mathrm{P}=0.004)$ and training $(\mathrm{P}=0.024)$ wraps.

In addition, there was a significant main effect for the duration of the ascent phase $(\mathrm{P} \leq 0.05$, $\left.\mathrm{p} \eta^{2}=0.35\right)$. Post-hoc pairwise comparisons showed that this duration was significantly greater in the nothing condition compared to the sleeve $(\mathrm{P}=0.003)$, competition wrap $(\mathrm{P}<0.001)$ and training wrap $(\mathrm{P}=0.005)$. There was a significant main effect for the percentage duration of the ascent phase $\left(\mathrm{P} \leq 0.05, \mathrm{p} \eta^{2}=0.35\right)$. Post-hoc pairwise comparisons showed that this duration was significantly greater in the nothing condition compared to the sleeve $(\mathrm{P}=0.01)$, competition wrap $(\mathrm{P}=0.002)$ and training wrap $(\mathrm{P}=0.01)$. In addition, it was also shown that percentage ascent phase duration was significantly greater in the knee sleeve compared to the competition wrap. A significant main effect for the percentage duration of the descent phase was also found $\left(\mathrm{P} \leq 0.05, \mathrm{p \eta}^{2}=0.35\right)$. Post-hoc pairwise comparisons showed that this duration was significantly greater in the sleeve $(\mathrm{P}=0.01)$, competition wrap $(\mathrm{P}=0.002)$ and training wrap $(\mathrm{P}=0.01)$ compared to the nothing condition. In addition it was also shown that percentage descent phase duration was significantly greater in the competition wrap compared to the knee sleeve $(\mathrm{P}=0.009)$.

There was also a main effect for the extent of anterior knee translation $\left(\mathrm{P} \leq 0.05, \mathrm{p} \eta^{2}=0.16\right)$. Post-hoc pairwise comparisons showed that knee translation was significantly greater in the nothing condition $(\mathrm{P}=0.02)$ compared to the competition wrap. Finally, there was a main effect for the extent of lateral knee displacement $\left(\mathrm{P} \leq 0.05, \mathrm{p} \eta^{2}=0.32\right)$. Post-hoc pairwise comparisons showed that lateral displacement was significantly greater in the nothing 
$(\mathrm{P}=0.03 \& \mathrm{P}=0.04)$ and sleeve $(\mathrm{P}=0.008 \& \mathrm{P}=0.002)$ conditions compared to the competition and training wraps.

\section{@@@TABLE1 NEARHERE@@@}

\section{Muscle forces}

There was a significant main effect for the integral of the quadriceps force during the ascent phase $\left(\mathrm{P} \leq 0.05, \mathrm{p \eta}^{2}=0.16\right)$. Post-hoc pairwise comparisons showed that the integral was significantly larger in the nothing condition $(\mathrm{P}=0.035)$ compared to the competition wrap. In addition, there was a significant main effect for the integral of the gluteus maximus force during the ascent phase $\left(\mathrm{P} \leq 0.05, \mathrm{p} \eta^{2}=0.18\right)$. Post-hoc pairwise comparisons showed that the gluteus maximus integral was significantly larger in the nothing condition $(\mathrm{P}=0.007)$ compared to the training wrap. There was also significant main effect for the integral of the hamstring force during the ascent phase $\left(\mathrm{P} \leq 0.05, \mathrm{p} \eta^{2}=0.18\right)$. Post-hoc pairwise comparisons showed that the hamstring integral was significantly larger in the nothing condition $(\mathrm{P}=0.018)$ compared to the training wrap. There was a significant main effect for the integral of the gastrocnemius force during the ascent phase $\left(\mathrm{P} \leq 0.05, \mathrm{p} \eta^{2}=0.26\right)$. Post-hoc pairwise comparisons showed that the gastrocnemius integral was significantly larger in the nothing $(\mathrm{P}=0.016)$ and sleeve $(\mathrm{P}=0.012)$ conditions compared to the competition wrap. Finally, there was a significant main effect for the integral of the soleus force during the ascent phase $\left(\mathrm{P} \leq 0.05, \mathrm{p}^{2}=0.25\right)$. Post-hoc pairwise comparisons showed that the soleus integral was significantly larger in the nothing $(\mathrm{P}=0.015)$ and sleeve $(\mathrm{P}=0.012)$ conditions compared to the competition wrap. 
Knee forces

326 There was a significant main effect for the peak knee shear force $\left(\mathrm{P} \leq 0.05, \mathrm{p} \eta^{2}=0.25\right)$. Posthoc pairwise comparisons showed that the peak shear force was significantly greater in the nothing $(\mathrm{P}=0.009)$ and knee sleeve $(\mathrm{P}=0.019)$ compared to the competition wrap condition.

\section{Kinematics}

There was a significant main effect for peak hip internal rotation $\left(\mathrm{P} \leq 0.05, \mathrm{p} \eta^{2}=0.39\right)$. Posthoc pairwise comparisons showed that peak internal rotation was significantly larger in the competition and training wraps compared to the nothing $(\mathrm{P}=0.001 \& \mathrm{P}=0.001)$ and knee sleeve conditions $(\mathrm{p}=0.019 \& \mathrm{p}=0.002)$.

There was a significant main effect for the sagittal plane knee $\operatorname{ROM}\left(P \leq 0.05, \mathrm{p} \eta^{2}=0.20\right)$. Post-hoc pairwise comparisons showed that ROM was significantly larger in the knee nothing condition compared to competition wrap $(\mathrm{P}=0.04)$ and in the knee sleeve in relation to the competition $(\mathrm{P}=0.03)$ and training wraps $(\mathrm{P}=0.004)$. There was also a significant main effect

342 for the peak knee adduction angle $\left(\mathrm{P} \leq 0.05, \mathrm{p} \eta^{2}=0.40\right)$. Post-hoc pairwise comparisons 
showed that peak knee adduction was significantly larger in the competition and training wraps compared to the nothing $(\mathrm{P}<0.001 \& \mathrm{P}=0.008)$ and knee sleeve conditions $(\mathrm{p}<0.001 \&$ $\mathrm{p}=0.005)$. There was also a main effect for the knee coronal plane $\operatorname{ROM}\left(\mathrm{P} \leq 0.05, \mathrm{p} \eta^{2}=0.37\right)$. Post-hoc pairwise comparisons showed that knee coronal plane ROM was significantly larger in the competition and training wraps compared to the nothing $(\mathrm{P}<0.001 \& \mathrm{P}=0.001)$ and knee sleeve conditions $(\mathrm{p}=0.013 \& \mathrm{p}=0.012)$.

There was a significant main effect for peak knee internal rotation $\left(\mathrm{P} \leq 0.05, \mathrm{p} \eta^{2}=0.31\right)$. Posthoc pairwise comparisons showed that peak internal rotation was significantly larger in the competition $(\mathrm{P}=0.001)$ and training $(\mathrm{P}<0001)$ wraps compared to the nothing condition. There was also a main effect for the knee transverse plane ROM $\left(\mathrm{P} \leq 0.05, \mathrm{p} \eta^{2}=0.28\right)$. Posthoc pairwise comparisons showed that knee transverse plane ROM was significantly larger in the competition $(\mathrm{P}=0.001)$ and training $(\mathrm{P}=0.001)$ wraps compared to the nothing condition, and in the training wrap $(\mathrm{P}=0.04)$ compared to the sleeve condition.

There was a significant main effect for peak ankle dorsiflexion $\left(\mathrm{P} \leq 0.05, \mathrm{p} \eta^{2}=0.23\right)$. Post-hoc pairwise comparisons showed that peak dorsiflexion was significantly larger in the nothing $(\mathrm{P}=0.001)$ and sleeve $(\mathrm{P}=0.005)$ conditions compared to the competition wrap. There was also a significant main effect for the sagittal plane ankle ROM $\left(P \leq 0.05, \mathrm{p} \eta^{2}=0.45\right)$. Post-hoc pairwise comparisons showed that sagittal plane ankle ROM was significantly larger in the nothing condition compared to the competition $(\mathrm{P}<0.001)$ and training wrap $(\mathrm{P}=0.03)$ and in the sleeve condition in relation to the competition wrap $(\mathrm{P}<0.001)$. 
366 There was a significant main effect for peak ankle eversion $\left(\mathrm{P} \leq 0.05, \mathrm{p} \eta^{2}=0.28\right)$. Post-hoc 367 pairwise comparisons showed that peak eversion was significantly larger in the sleeve

\section{@@@TABLE4 NEARHERE@@@}

\section{Subjective ratings}

For the subjectively preferred condition 7 participants selected the sleeve, 3 the nothing condition, 3 the training wrap and 2 the competition wrap. The chi-squared test was significant $\left(X^{2}=3.93, \mathrm{P}<0.05\right)$ and indicated that there was a preference towards the sleeve condition. For the subjective ratings of comfort in the sleeve, 9 participants rated that this condition improved comfort, 4 no-change and 2 reduced comfort. The chi-squared test was significant $\left(X^{2}=5.20, \mathrm{P}<0.05\right)$ and significantly more participants found that the sleeve provided improved comfort. For the ratings of knee stability in the sleeve, 10 participants rated that this condition improved stability, 3 no-change and 2 reduced stability. The chisquared test was significant $\left(X^{2}=7.60, \mathrm{P}<0.05\right)$ and significantly more participants found that the sleeve provided improved stability. For the subjective ratings of comfort in the training wrap, 2 participants rated that this condition improved comfort, 3 no-change and 10 reduced comfort. The chi-squared test was significant $\left(X^{2}=7.60, \mathrm{P}<0.05\right)$ and showed that 
significantly more participants found that the training wrap reduced comfort. For the ratings of knee stability in the training wrap, 9 participants rated that this condition improved stability, 4 no-change and 2 reduced stability. The chi-squared test was significant $\left(X^{2}=5.20\right.$, $\mathrm{P}<0.05)$ and significantly more participants found that the training wrap provided improved stability. For the subjective ratings of comfort in the competition wrap, 2 participants rated that this condition improved comfort, 4 no-change and 9 reduced comfort. The chi-squared test was significant $\left(X^{2}=5.20, \mathrm{P}<0.05\right)$ and showed that significantly more participants found that the competition wrap reduced comfort. For the ratings of knee stability in the competition wrap, 11 participants rated that this condition improved stability, 2 no-change and 2 reduced stability. The chi-squared test was significant $\left(X^{2}=10.80, \mathrm{P}<0.05\right)$ and significantly more participants found that the competition wrap provided improved stability.

\section{Discussion}

The aim of the current investigation was to comparatively examine the effects of knee wraps/ sleeves on kinetics, three-dimensional kinematics and muscle forces during the squat. To the authors knowledge this investigation represents the first to explore the aforementioned aims and may provide further insight regarding the effects of knee wraps/ sleeves on the mechanics of the barbell back squat.

Previous analyses have shown that knee wraps influence performance parameters during the back squat. Specifically, Lake et al., (3) showed that knee wraps significantly enhanced mechanical power output during the ascent phase of the lift. The findings from the current investigation do not support these observations as no significant alterations in power output 
or GRF parameters during the ascent phase were evident as a function of wearing knee

413 wraps/ sleeves. Similarly, Lake et al., (3) showed that the lowering phase was performed

414 faster when knee wraps were worn, allowing elastic potential energy to be stored within the

415 knee wraps, increasing the vertical force applied to the centre of mass and augmenting the

416 power output during the ascent phase. The findings from this investigation do not agree with

417 those of Lake et al, (3), as the knee sleeve/ wraps increased the descent phase and decreased

418 the ascent phase duration, which may serve as the mechanical explanation for the lack of

419 improvements in performance parameters. The lack of agreement between analyses may be

420 due to the lower relative and absolute mass being lifted, alongside the participants' lack of

421 familiarity in using knee wraps/ sleeves. In contrast to the current study, in the investigation

of Lake et al., (3), athletes lifted at $80 \%$ of $1 \mathrm{RM}$ relative to a group maximum squat capacity

of $160.5 \mathrm{~kg}$ and had previous experience of squatting using knee wraps. The findings from

the current investigation therefore indicate that knee wraps/ sleeves may not mediate

improvements in performance parameters when lower masses are being lifted, in athletes who

are not accustomed to using them. This leads to the notion that the mechanical effects of knee

wraps/ sleeves may be mass (lifted) and experience dependant, and this is something that

future research should seek to full substantiate.

Importantly, the current investigation did show that muscle force parameters were significantly influenced by the experimental conditions. Specifically, knee wraps statistically reduced the integral of each muscle group during the ascent phase compared to the nothing condition, and in the gastrocnemius and soleus muscles in relation to the knee sleeve. This observation supports the findings of Gomes et al., (6) who showed using EMG that knee wraps statistically influenced muscle outputs during the ascent phase, and also the proposition suggested by Lake et al., (3) that knee wraps may affect the target musculature. 
437 Gomes et al., (6) hypothesized that reductions in vastus lateralis muscle recruitment were 438 initiated by tissue pressure imposed by the knee wrap, leading to inhibition of the muscle 439 motoneuron pool. However, the current investigation indicates that this may not be the case, 440 as reductions were found in musculature that does not directly interface with the knee wraps. 441 It is proposed that the aforementioned reductions in muscle kinetics were mediated by carryover (5). Muscle force attenuation in the knee wrap/ sleeve conditions was due (in spite

of the same absolute load being lifted) to the lifters operating at a lower relative intensity

compared to squatting without external aid. This indicates that lifters who utilize knee wraps/

sleeves may be able to lift greater maximal loads during competition or perform additional

repetitions with a given load. Nonetheless, mechanical tension is the primary driver of muscle

hypertrophy (1) and the cross-sectional area is the key determiner of muscle force production

(25). As such, skeletal muscle training impulses determine the magnitude of adaptive

hypertrophic and performance responses (26). Therefore, as knee wraps significantly reduced

lower extremity muscular recruitment during the ascent phase, this indicates that their

utilization in relation to the nothing and (to a lesser extent) knee sleeve conditions may not be

advisable in athletes seeking to maximise training adaptations.

In agreement with the findings of Lake et al., (3) this study showed that knee wraps significantly altered movement patterns during the back squat exercise, in relation to squatting in the nothing condition. Importantly, sagittal plane knee ROM and the anterior knee translation were statistically reduced in the knee wraps compared to the nothing condition. It is likely that the reduced knee translation/ flexion ROM were responsible for the reductions in horizontal bar displacement that were similarly shown in the knee wrap

460 conditions. Similar to Lake et al., (3) this observation is supported by the anterior-posterior 461 GRF integral during the descent phase, which was to be posteriorly orientated in both knee 
wraps but directed anteriorly in the nothing condition and knee sleeve. The above observations are supported by the subjective ratings of the knee wrap conditions, which indicate that knee stability was significantly enhanced but with corresponding reductions in perceived comfort. The above observations reinforce the propositions of both Lake et al., (3) and Gomes et al., (6) who postulated that the discomfort mediated by knee wraps creates a physical barrier about the knee joint. From and injury prevention perspective it could nonetheless be interpreted that the decreases in anterior knee translation were important given the attenuation of the peak knee shear force when wearing knee wraps. However, taking into account knee wraps potential to diminish lower extremity muscle development and alter natural squatting mechanics; further analyses are required before this could be properly established.

In addition to the above, it was also revealed that both coronal and transverse plane hip and knee kinematics were significantly influenced by the competition and training knee wrap conditions. This observation was likely mediated by the reductions in lateral knee displacement that were observed when wearing knee wraps and reinforces the Lake et al., (3) and Gomes et al., (6) notion in relation to the physical restriction about the knee joint. In conjunction with the results outlined previously, this finding provides further evidence to show that knee wraps influence natural squatting mechanics as differences in relation to the nothing condition were observed all three planes of rotation. Finally, like the knee wrap conditions the knee sleeve did not mediate improvements in mechanical power output and statistically influenced the duration of the different phases of the squat. However, unlike the knee wraps the knee sleeves did not significantly alter natural 
squatting mechanics or influence muscle kinetics during the ascent phase in relation to the

487 nothing condition. It is proposed that this observation was mediated by the significant improvements in both perceived comfort and stability that were noted in the knee sleeves in relation to the nothing condition. Therefore, taking the above into account and the subjective

490 preference towards this condition, the findings from the current investigation indicate that knee sleeves may be more efficacious for athletes who regularly utilize the back squat for their training goals, although future longitudinal studies are required before this can be fully substantiated.

A potential drawback to the current investigation is that only recreational lifters were examined as part of the current study. Previous analyses have shown that squat experience can significantly influence the biomechanics of performing the squat itself (27). Therefore, it a more experienced group of lifters.

In conclusion, the effects of knee wraps/ sleeves on the biomechanics of the barbell back squat have received limited research attention. Therefore, the present study adds to the current scientific knowledge, by providing a comprehensive evaluation regarding the effects of knee wraps/ sleeves on kinetics, three-dimensional kinematics and muscle forces during 507 the squat. Importantly, knee wraps significantly reduced lower extremity muscle integrals during the ascent phase, natural squatting mechanics in all three planes of rotation and also reduced perceived comfort. However, knee sleeves were conversely able to mediate 
510

511 alter natural squatting mechanics or influence muscle kinetics during the ascent phase.

512 Taking into account the potential of knee wraps to diminish lower extremity muscle

513 development; knee sleeves may be more efficacious for athletes who regularly utilize the

514 back squat for their training goals, although further longitudinal analyses are required before 515 this can be fully established.

516

517

518

519

520

521

522

523

524

525

526

527

528

529

530

531

\section{Acknowledgments}

The authors would like to thank Strength Shop Ltd, UK who donated the knee sleeves used in this investigation and also SBD Apparel Ltd, UK who provided the knee wraps utilized in this study. However, the results of this study do not constitute endorsement by the authors.

\section{References}

1. Schoenfeld, B. J. (2010). Squatting kinematics and kinetics and their application to exercise performance. The Journal of Strength \& Conditioning Research, 24(12), 3497-3506.

2. Paoli, A., Marcolin, G., \& Petrone, N. (2009). The effect of stance width on the electromyographical activity of eight superficial thigh muscles during back squat with different bar loads. The Journal of Strength \& Conditioning Research, 23(1), 246-250.

3. Lake, J. P., Carden, P. J., \& Shorter, K. A. (2012). Wearing knee wraps affects mechanical output and performance characteristics of back squat exercise. The Journal of Strength \& Conditioning Research, 26(10), 2844-2849. 

A., \& Gomes, W. A. (2015). Can the technique of knee wrap placement affect the maximal isometric force during back squat exercise. Int J Sports Sci, 5(1), 16-18.

5. Gomes, W. A., Serpa, E. P., Soares, E. G., da Silva, J. J., Corrêa, D. A., de Oliveira, F. H. D., \& Marchetti, P. H. (2014). Acute effects on maximal isometric force with and without knee wrap during squat exercise. Int J Sports Sci, 4(2), 47-9.

6. Gomes, W. A., Brown, L. E., Soares, E. G., da Silva, J. J., Fernando, H. D. O., Serpa, É. P., \& Marchetti, P. H. (2015). Kinematic and sEMG analysis of the back squat at different intensities with and without knee wraps. The Journal of Strength \& Conditioning Research, 29(9), 2482-2487.

7. Sinclair, J. K., Vincent, H., \& Richards, J. D. (2017). Effects of prophylactic knee bracing on knee joint kinetics and kinematics during netball specific movements. Physical Therapy in Sport, 23, 93-98.

8. Cappozzo, A., Catani, F., Della Croce, U., \& Leardini, A. (1995). Position and orientation in space of bones during movement: anatomical frame definition and determination. Clinical biomechanics, 10(4), 171-178.

9. Sinclair, J. K., Brooks, D., \& Atkins, S. (2017). An examination of the hamstring and the quadriceps muscle kinematics during the front and back squat in males. Baltic Journal of Health and Physical Activity, 9(1), 37-45.

10. Graydon, R. W., Fewtrell, D. J., Atkins, S., \& Sinclair, J. K. (2015). The test-retest reliability of different ankle joint center location techniques. Foot and ankle online journal, 1(11), 10-15.

11. Sinclair, J., Taylor, P. J., Currigan, G., \& Hobbs, S. J. (2014). The test-retest reliability of three different hip joint centre location techniques. Movement \& Sport Sciences-Science \& Motricité, (83), 31-39. 
12. Sinclair, J., Hebron, J., \& Taylor, P. J. (2015). The test-retest reliability of knee joint center location techniques. Journal of Applied Biomechanics, 31(2), 117-121.

13. Lahti, J., Hegyi, A., Vigotsky, A. D., \& Ahtiainen, J. P. (2019). Effects of barbell back squat stance width on sagittal and frontal hip and knee kinetics. Scandinavian journal of medicine \& science in sports, 29(1), 44-54.

14. Sinclair, J., McCarthy, D., Bentley, I., Hurst, H. T., \& Atkins, S. (2015). The influence of different footwear on 3-D kinematics and muscle activation during the barbell back squat in males. European journal of sport science, 15(7), 583-590.

15. Sinclair, J. K., Atkins, S. J., Kudiersky, N., Taylor, P. J., \& Vincent, H. (2015). Effects of front and back squat techniques on patellofemoral joint kinetics in males. Journal of Biomedical Engineering and Informatics, 2(1), 76-81.

16. Van Eijden, T. M. G. J., Kouwenhoven, E., Verburg, J., \& Weijs, W. A. (1986). A mathematical model of the patellofemoral joint. Journal of biomechanics, 19(3), 219229.

17. Willson, J. D., Sharpee, R., Meardon, S. A., \& Kernozek, T. W. (2014). Effects of step length on patellofemoral joint stress in female runners with and without patellofemoral pain. Clinical biomechanics, 29(3), 243-247.

18. Ward, S. R., Eng, C. M., Smallwood, L. H., \& Lieber, R. L. (2009). Are current measurements of lower extremity muscle architecture accurate?. Clinical orthopaedics and related research, 467(4), 1074-1082.

19. Németh, G., \& Ohlsén, H. (1985). In vivo moment arm lengths for hip extensor muscles at different angles of hip flexion. Journal of biomechanics, 18(2), 129-140.

20. Self, B. P., \& Paine, D. (2001). Ankle biomechanics during four landing techniques. Medicine and science in sports and exercise, 33(8), 1338-1344. 
21. Sinclair, J., Atkins, S., \& Vincent, H. (2014). Influence of different hip joint centre locations on hip and knee joint kinetics and kinematics during the squat. Journal of human kinetics, 44(1), 5-17.

22. Janssen, I., Steele, J. R., Munro, B. J., \& Brown, N. A. (2013). Predicting the patellar tendon force generated when landing from a jump. Medicine and Science in Sports and Exercise, 45(5), 927-934.

23. Herzog, W., \& Read, L. J. (1993). Lines of action and moment arms of the major force-carrying structures crossing the human knee joint. Journal of Anatomy, 182(Pt 2), 213.

24. Besier, T. F., Draper, C. E., Gold, G. E., Beaupré, G. S., \& Delp, S. L. (2005). Patellofemoral joint contact area increases with knee flexion and weight-bearing. Journal of Orthopaedic Research, 23(2), 345-350.

25. Vigotsky, A. D., Contreras, B., \& Beardsley, C. (2015). Biomechanical implications of skeletal muscle hypertrophy and atrophy: a musculoskeletal model. Peer J, 3, e1462.

26. Winwood, P.W., Keogh, J.W., \& Harris, N.K. (2012). Interrelationships between strength, anthropometrics, and strongman performance in novice strongman athletes. The Journal of Strength \& Conditioning Research, 26(2), 513-522.

27. Lorenzetti, S., Ostermann, M., Zeidler, F., Zimmer, P., Jentsch, L., List, R., \& Schellenberg, F. (2018). How to squat? Effects of various stance widths, foot placement angles and level of experience on knee, hip and trunk motion and loading. BMC Sports Science, Medicine and Rehabilitation, 10(1), 14-19. 
Table 1: Kinetic and temporal parameters (Mean $\pm S D)$ as a function of each experimental condition.

\begin{tabular}{|c|c|c|c|c|c|c|c|c|c|}
\hline & \multicolumn{2}{|c|}{ Nothing } & \multicolumn{2}{|c|}{ Sleeve } & \multicolumn{2}{|c|}{$\begin{array}{c}\text { Competition } \\
\text { wrap }\end{array}$} & \multicolumn{2}{|c|}{ Training wrap } & \\
\hline & Mean & $S D$ & Mean & $S D$ & Mean & $S D$ & Mean & $S D$ & \\
\hline Peak bar velocity $(\mathrm{m} / \mathrm{s})$ & 1.01 & 0.14 & 1.11 & 0.37 & 1.05 & 0.17 & 1.05 & 0.18 & \\
\hline Anterior bar displacement (m) & 0.09 & 0.03 & 0.08 & 0.03 & 0.07 & 0.02 & 0.08 & 0.02 & \\
\hline Total duration (s) & 2.60 & 0.36 & 2.56 & 0.39 & 2.59 & 0.42 & 2.53 & 0.45 & \\
\hline Ascent duration (s) & $1.33 \mathrm{ABC}$ & 0.20 & 1.27 & 0.21 & 1.21 & 0.17 & 1.22 & 0.19 & $*$ \\
\hline Descent duration (s) & 1.27 & 0.26 & 1.29 & 0.29 & 1.38 & 0.32 & 1.31 & 0.32 & \\
\hline Ascent percent duration (\%) & $\begin{array}{l}51.35 \\
A B C\end{array}$ & 5.20 & 49.91 & 5.64 & 47.56 & 5.73 & 48.72 & 5.14 & * \\
\hline Descent percent duration (\%) & $\begin{array}{l}48.65 \\
A B C\end{array}$ & 5.20 & 50.09 & 5.64 & 52.44 & 5.73 & 51.28 & 5.14 & * \\
\hline Knee anterior translation $(\mathrm{cm})$ & $20.50 \mathrm{~B}$ & 2.87 & 20.49 & 3.56 & 19.07 & 4.06 & 19.93 & 4.45 & * \\
\hline Knee lateral translation $(\mathrm{cm})$ & $13.41 B C$ & 3.04 & $13.85 B C$ & 3.53 & 12.29 & 2.88 & 12.51 & 3.06 & $*$ \\
\hline Peak vertical force $(\mathrm{N} / \mathrm{kg})$ & 12.80 & 2.06 & 13.19 & 1.77 & 12.83 & 1.45 & 13.19 & 1.69 & \\
\hline $\mathrm{RFD}(\mathrm{N} / \mathrm{kg} / \mathrm{s})$ & 68.51 & 23.85 & 64.79 & 20.01 & 65.89 & 24.98 & 63.67 & 21.11 & \\
\hline Medial GRF integral ascent (N/kg·s) & 1.80 & 0.81 & 1.74 & 0.76 & 1.84 & 0.81 & 1.68 & 0.74 & \\
\hline Posterior GRF integral ascent (N/kg.s) & 0.04 & 0.12 & 0.04 & 0.13 & 0.02 & 0.13 & 0.02 & 0.16 & \\
\hline Vertical GRF integral ascent (N/kg.s) & 13.09 & 3.28 & 12.83 & 2.61 & 12.33 & 2.60 & 12.38 & 2.91 & \\
\hline Medial GRF integral descent (N/kg.s) & 1.43 & 0.68 & 1.50 & 0.71 & 1.88 & 0.89 & 1.60 & 0.80 & \\
\hline Posterior GRF integral descent (N/kg.s) & -0.02 & 0.08 & -0.02 & 0.09 & 0.02 & 0.11 & 0.01 & 0.13 & \\
\hline Vertical GRF integral descent (N/kg·s) & $12.61 \mathrm{~A}$ & 2.91 & 13.00 & 2.70 & $14.17 \mathrm{~A}$ & 3.69 & 13.47 & 3.77 & $*$ \\
\hline Peak knee shear force (N/kg) & 7.68 & 2.15 & 7.62 & 2.09 & 6.90 & 1.82 & 7.57 & 2.21 & \\
\hline Peak power (W/kg) & 20.21 & 4.58 & 19.55 & 3.94 & 19.73 & 2.95 & 20.84 & 4.05 & \\
\hline Stance width $(\mathrm{m})$ & 0.49 & 0.06 & 0.49 & 0.06 & 0.50 & 0.06 & 0.49 & 0.05 & \\
\hline
\end{tabular}

Key: * = significant main effect

$\boldsymbol{A}=$ significantly different from Sleeve

$\boldsymbol{B}=$ significantly different from Competition wrap

$\boldsymbol{C}=$ significantly different from Training wrap 
Table 2: Muscle forces (Mean \pm SD) as a function of each experimental condition.

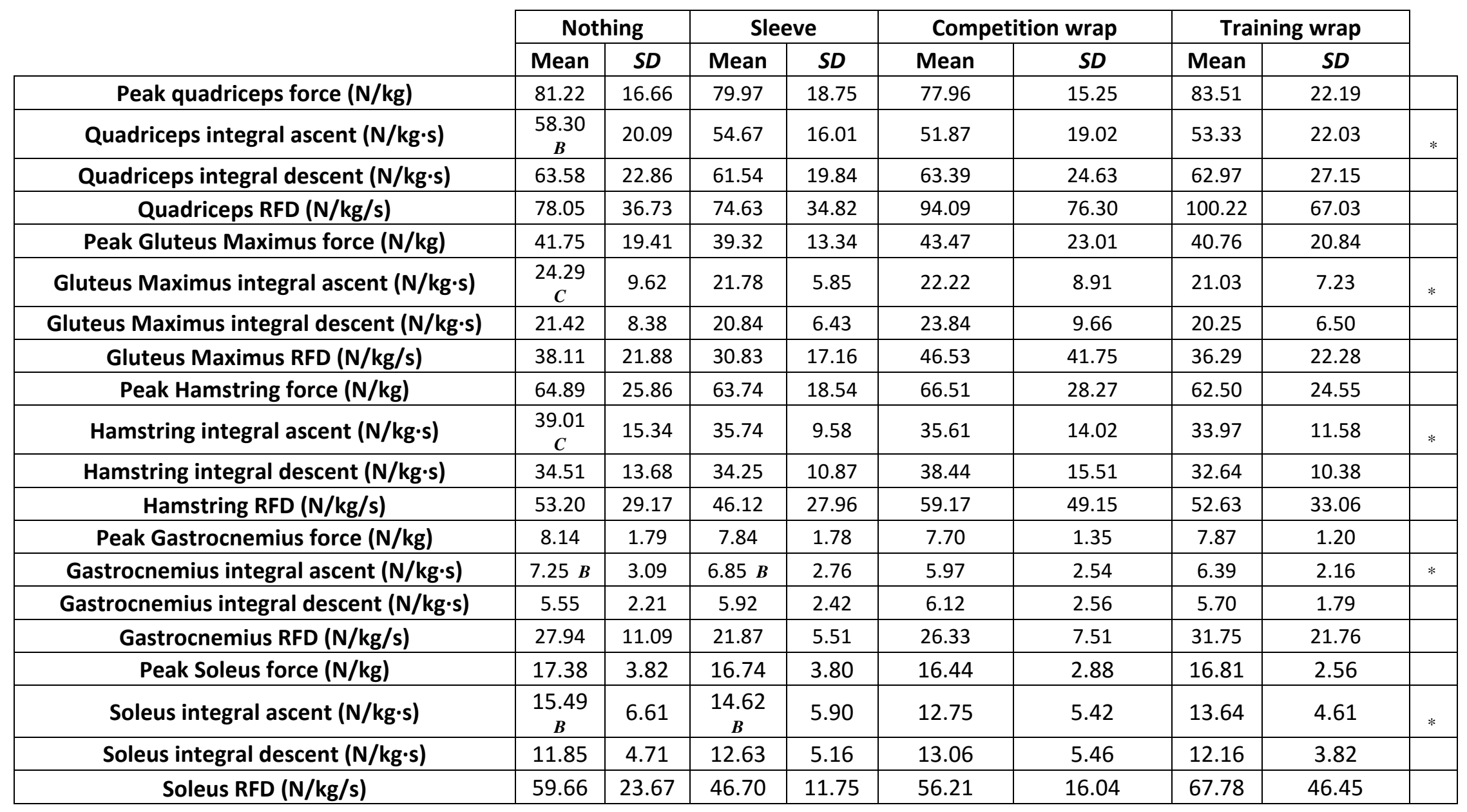

Key: $*$ s significant main effect

$\boldsymbol{A}=$ significantly different from Sleeve

$\boldsymbol{B}=$ significantly different from Competition wrap

$C=$ significantly different from Training wrap 
Table 3: Knee forces (Mean $\pm S D)$ as a function of each experimental condition.

\begin{tabular}{|c|c|c|c|c|c|c|c|c|c|}
\hline & \multicolumn{2}{|c|}{ Nothing } & \multicolumn{2}{|c|}{ Sleeve } & \multicolumn{2}{|c|}{$\begin{array}{c}\text { Competition } \\
\text { wrap }\end{array}$} & \multicolumn{2}{|c|}{ Training wrap } & \\
\hline & Mean & $S D$ & Mean & $S D$ & Mean & $S D$ & Mean & $S D$ & \\
\hline Peak knee shear force (N/kg) & $7.68 B$ & 2.15 & 7.62 B & 2.09 & 6.90 & 1.82 & 7.25 & 2.20 & $*$ \\
\hline Knee shear force integral (N/kg.s) & 12.31 & 5.15 & 12.01 & 4.67 & 11.34 & 4.93 & 11.77 & 5.51 & \\
\hline Knee shear force instantaneous load rate $(\mathrm{N} / \mathrm{kg} / \mathrm{s})$ & 30.03 & 10.63 & 29.68 & 7.80 & 26.80 & 7.83 & 28.73 & 9.91 & \\
\hline Peak patellar tendon force (N/kg) & 62.08 & 21.50 & 63.34 & 22.50 & 57.91 & 20.03 & 64.70 & 25.89 & \\
\hline Patellar tendon force integral (N/kg.s) & 85.47 & 35.29 & 81.28 & 28.93 & 79.45 & 35.62 & 84.09 & 44.75 & \\
\hline Patellar tendon force instantaneous load rate $(\mathrm{N} / \mathrm{kg} / \mathrm{s})$ & 264.35 & 99.95 & 261.90 & 77.17 & 240.70 & 84.61 & 258.67 & 94.49 & \\
\hline Peak patellofemoral force (N/kg) & 46.78 & 10.68 & 46.81 & 12.02 & 45.54 & 9.67 & 49.22 & 14.14 & \\
\hline Patellofemoral force integral (N/kg.s) & 67.93 & 24.03 & 65.27 & 18.69 & 64.44 & 25.01 & 66.19 & 29.46 & \\
\hline Patellofemoral force instantaneous load rate $(\mathrm{N} / \mathrm{kg} / \mathrm{s})$ & 196.02 & 68.09 & 177.75 & 46.28 & 167.43 & 54.13 & 187.48 & 71.96 & \\
\hline Patellofemoral tendon stress (KPa/kg) & 58.50 & 13.35 & 57.76 & 13.63 & 56.52 & 12.12 & 60.51 & 17.30 & \\
\hline Patellofemoral stress integral (KPa/kg·s) & 88.90 & 31.29 & 85.31 & 23.93 & 84.63 & 32.23 & 87.31 & 38.94 & \\
\hline Patellofemoral stress instantaneous load rate $(\mathrm{KPa} / \mathrm{kg} / \mathrm{s})$ & 298.41 & 108.48 & 284.00 & 82.99 & 272.84 & 106.87 & 291.39 & 99.66 & \\
\hline
\end{tabular}

Key: $*$ significant main effect

$\boldsymbol{A}=$ significantly different from Sleeve

$\boldsymbol{B}=$ significantly different from Competition wrap

$\boldsymbol{C}=$ significantly different from Training wrap

Table 4: Kinematic parameters (Mean $\pm S D$ ) as a function of each experimental condition. 


\begin{tabular}{|c|c|c|c|c|c|c|c|c|c|}
\hline \multirow[b]{2}{*}{ Trunk (Sagittal plane) } & \multicolumn{2}{|c|}{ Nothing } & \multicolumn{2}{|c|}{ Sleeve } & \multicolumn{2}{|c|}{ Competition wrap } & \multicolumn{2}{|c|}{ Training wrap } & \\
\hline & Mean & $S D$ & Mean & $S D$ & Mean & $S D$ & Mean & $S D$ & \\
\hline Peak flexion $\left({ }^{\circ}\right)$ & 38.58 & 6.72 & 37.82 & 6.85 & 38.01 & 6.14 & 37.85 & 6.01 & \\
\hline ROM $\left({ }^{\circ}\right)$ & 28.19 & 3.90 & 27.62 & 4.78 & 27.29 & 4.38 & 27.55 & 4.54 & \\
\hline \multicolumn{10}{|l|}{ Hip (Sagittal plane $+=$ flexion) } \\
\hline Peak flexion $\left({ }^{\circ}\right)$ & 106.70 & 19.15 & 107.14 & 18.15 & 106.50 & 16.76 & 103.81 & 19.32 & \\
\hline $\operatorname{ROM}\left({ }^{\circ}\right)$ & 87.38 & 18.15 & 92.39 & 14.48 & 86.19 & 14.82 & 89.73 & 15.62 & \\
\hline \multicolumn{10}{|l|}{ Hip (Coronal plane $+=$ adduction) } \\
\hline Peak abduction $\left({ }^{\circ}\right)$ & -29.07 & 8.25 & -30.80 & 7.76 & -29.56 & 5.72 & -30.08 & 7.89 & \\
\hline $\operatorname{ROM}\left({ }^{\circ}\right)$ & 18.52 & 8.46 & 20.79 & 7.60 & 18.72 & 6.21 & 18.94 & 8.26 & \\
\hline \multicolumn{10}{|c|}{ Hip (Transverse plane $+=$ internal rotation) } \\
\hline Peak internal rotation $\left({ }^{\circ}\right)$ & $10.80 B C$ & 13.19 & $11.50 \mathrm{BC}$ & 13.44 & 18.78 & 11.21 & 21.19 & 9.29 & * \\
\hline $\operatorname{ROM}\left({ }^{\circ}\right)$ & 26.48 & 10.33 & 27.67 & 9.64 & 24.72 & 8.26 & 29.63 & 10.97 & \\
\hline \multicolumn{10}{|l|}{ Knee (Sagittal plane + = flexion) } \\
\hline Peak flexion $\left({ }^{\circ}\right)$ & 117.76 & 15.88 & 117.27 & 14.94 & 114.06 & 14.47 & 115.58 & 15.80 & \\
\hline $\operatorname{ROM}\left({ }^{\circ}\right)$ & 109.57 & 14.25 & 111.14 & 13.29 & 105.96 & 14.39 & 107.41 & 15.30 & * \\
\hline \multicolumn{10}{|c|}{ Knee (Coronal plane $+=$ adduction) } \\
\hline Peak adduction $\left({ }^{\circ}\right)$ & $8.64 B C$ & 5.38 & $9.27 B C$ & 6.86 & 17.65 & 6.76 & 17.44 & 6.55 & * \\
\hline $\operatorname{ROM}\left({ }^{\circ}\right)$ & $6.87 B C$ & 4.25 & $7.41 B C$ & 5.64 & 14.81 & 7.25 & 15.03 & 6.51 & $*$ \\
\hline \multicolumn{10}{|c|}{ Knee (Transverse plane $+=$ internal rotation) } \\
\hline Peak internal rotation $\left({ }^{\circ}\right)$ & $19.81 B C$ & 9.32 & 24.26 & 15.79 & 31.45 & 12.70 & 29.62 & 10.59 & * \\
\hline $\operatorname{ROM}\left({ }^{\circ}\right)$ & $22.95 B C$ & 11.61 & $24.86 C$ & 18.82 & 34.17 & 12.41 & 33.12 & 10.59 & * \\
\hline \multicolumn{10}{|c|}{ Ankle (Sagittal plane $+=$ dorsiflexion) } \\
\hline Peak dorsiflexion $\left({ }^{\circ}\right)$ & $27.72 B$ & 5.65 & $27.46 B$ & 6.04 & 23.96 & 5.98 & 25.91 & 7.29 & * \\
\hline ROM ( $\left.{ }^{\circ}\right)$ & $28.29 B C$ & 5.64 & $27.89 B$ & 5.76 & 24.04 & 6.55 & 26.28 & 6.68 & * \\
\hline \multicolumn{10}{|c|}{ Ankle (Coronal plane $+=$ inversion) } \\
\hline Peak eversion $\left({ }^{\circ}\right)$ & $-9.14 A B C$ & 5.13 & -11.43 & 6.90 & -14.31 & 7.13 & -12.23 & 4.84 & * \\
\hline $\operatorname{ROM}\left({ }^{\circ}\right)$ & $9.25 B C$ & 4.28 & 11.08 & 5.61 & 12.72 & 4.81 & 12.38 & 3.53 & * \\
\hline \multicolumn{10}{|c|}{ Ankle (Transverse plane $+=$ internal rotation) } \\
\hline Peak external rotation $\left({ }^{\circ}\right)$ & -6.36 & 5.10 & -4.74 & 4.00 & -4.95 & 5.31 & -3.52 & 5.62 & \\
\hline $\operatorname{ROM}\left({ }^{\circ}\right)$ & 8.34 & 4.42 & 7.14 & 4.56 & 8.02 & 5.09 & 6.89 & 4.14 & \\
\hline
\end{tabular}


Key: * significant main effect

$\boldsymbol{A}=$ significantly different from Sleeve

$\boldsymbol{B}=$ significantly different from Competition wrap

$\boldsymbol{C}=$ significantly different from Training wrap 